\title{
El sistema de la Integración Centroamericana (SICA) y la tutela de los Derechos Humanos ${ }^{1}$
}

\author{
The Central American Integration System (SICA) \\ and the protection of human rights
}

\author{
Diana Cecilia Santana Paisano²
}

Resumen: Los compromisos adquiridos por el SICA respecto de la defensa de los Derechos Humanos están consignados en todo su entramado normativo e institucional, que abarca desde los tratados fundacionales de la integración centroamericana y los acuerdos de Esquipulas (que plantearon una visión renovada del modelo de integración en función de la democracia y la seguridad humana), pasando por el amplísimo conjunto de derechos reconocidos por el sistema interamericano que confiere jurisdicción a la Corte Interamericana de Derechos Humanos (Corte IDH), hasta el propio orden universal procedente de la Organización de las Naciones Unidas (ONU), reforzándose de esta manera como eslabones de protección y justicia universal, y constituyéndose también como los principios base que pueden ser invocados de forma similar ante la Corte Centroamericana de Justicia (CCJ), pues estos principios forman parte del propio Derecho Comunitario Centroamericano, aunque existen limitaciones en cuanto a las competencias judiciales específicas de la CCJ.

Palabras clave: SICA, integración regional, derechos humanos, ius standi, principio pro homie, tutela judicial.

Abstract: The commitments acquired by SICA with respect to the defense of Human Rights are recorded throughout its normative and institutional framework, which includes the founding treaties of Central American integration as well as the Esquipulas agreements (which raised a renewed vision of the integration model based on democracy and human security), the wide range of rights recognized by the inter-American system that confers jurisdiction to the Inter-American Court of Human Rights (Court HDI), and the universal order established by the United Nations Organization (UN). This has thus reinforced the commitments to affording protection and universal justice and it has also turned them into the basic principles to be invoked before the Central American Court of Justice (CACJ), because these principles are part of the Central American Community Law itself, despite limitations regarding the specific judicial powers of the CACJ.

Keywords: SICA, regional integration, human rights, ius standi, pro homie principle, judicial protection.

\footnotetext{
Doi: https://doi.org/10.24215/23142766e079

${ }^{1}$ Recibido el 14/08/2019. Aceptado el 30/11/2019

2 Profesora de la Facultad de Ciencias Jurídicas, Universidad Centroamericana (UCA) de Managua, Nicaragua. Máster regional en integración centroamericana y desarrollo. Doctoranda en Desarrollo inclusive y sostenible. Universidad Loyola Andalucía. España. E-mail: dsantanapaisano@yahoo.com
} 


\section{Introducción}

Centroamérica, una región cuyo proceso de integración data de más de 60 años de existencia, combina una diversidad de potencialidades y desafíos en torno a su desarrollo humano. Las desigualdades socioeconómicas y los conflictos políticos hacen que los académicos tengamos en permanente estudio las situaciones que impiden a los ocho países adheridos a un proceso de integración denominado Sistema de la Integración Centroamericana (SICA) alcanzar los objetivos que se han propuesto desde 1950 y que han renovado en diversas ocasiones, en pos de ese anhelado desarrollo humano.

Un somero balance de corto plazo nos hace caer en la cuenta de que Centroamérica ha acumulado un decenio de relativa estabilidad económica, que no necesariamente se traduce en la superación de problemas estructurales de carácter social y humano, habida cuenta de que en el año próximo pasado podemos remembrar los casos de Costa Rica, con las protestas sociales a causa de la subida de tributos al consumo y la renta ${ }^{3}$; El Salvador, con el contexto electoral que devino en un giro sin precedentes sobre los resultados electorales de voto castigo a los partidos tradicionales ${ }^{4}$; Honduras, que aún se encuentra recuperándose del conflicto social a causa de serios señalamientos de fraude electoral, con fuertes acusaciones de represión gubernamental ${ }^{5}$; Guatemala, con los conflictos surgidos ante las acciones gubernamentales para la expulsión de la misión de la Comisión Internacional Contra la Impunidad en Guatemala6; Panamá, con las protestas estudiantiles por una propuesta de reforma a la seguridad social que no prosperó7; Nicaragua, con un conflicto sociopolítico que ha perdurado ante la represión gubernamental, aunado a graves heridas reabiertas por la comisión de delitos de lesa humanidad en contra de población civil $;$ y, cómo olvidar las caravanas de migrantes hondureños y guatemaltecos -en su mayoría- dirigiéndose hacia Estados Unidos ${ }^{9}$, por mencionar algunos, los más mediatizados.

Estas situaciones, como vemos, están vinculadas con los derechos humanos, desde los más primarios como la vida y la libertad, pasando por los derechos políticos como la democracia, hasta los derechos económicos y sociales, de primera, segunda y tercera generación, como la academia los clasifica.

Ante este escenario cabe preguntarnos, siendo los Estados miembros del SICA parte de los sistemas universal e interamericano de protección de $\mathrm{DDHH}$, si existe algún marco de actuación de esta organización regional que coadyuve a los esfuerzos de los demás organismos internacionales de protección de los derechos humanos. En este artículo esbozaremos los elementos que nos permiten dar una respuesta afirmativa a este cuestionamiento, ma-

\footnotetext{
${ }^{3}$ https://www.prensa-latina.cu/index.php?o=rn\&id=287701\&SEO=nuevos-impuestos-en-costa-rica-en-medio-de-protestas

${ }^{4}$ https://cnnespanol.cnn.com/2019/02/03/elecciones-el-salvador-salvadorenos-eligen-presidente-nuevo/

${ }^{5}$ https://www.bbc.com/mundo/noticias-america-latina-48506352

${ }^{6}$ https://www.bbc.com/mundo/noticias-america-latina-46790805

${ }^{7}$ https://elpais.com/internacional/2018/04/19/america/1524095967_183633.html

${ }^{8}$ https://www.dw.com/es/claves-para-entender-un-a\%C3\%B1o-de-crisis-en-nicaragua/a-48357261

${ }^{9}$ https://elpais.com/internacional/2019/04/10/america/1554918875_800216.html
} 
tizando las limitaciones jurídicas que el propio sistema posee, en cuanto a su cuerpo normativo y el ámbito de actuaciones competenciales de sus instituciones regionales.

\section{Estructura del derecho comunitario centroamericano}

Para iniciar, debemos partir de la consideración de que el Sistema de la Integración Centroamericana (SICA) es una organización regional de Estados con una institucionalidad que tiene su base jurídica en el denominado Derecho Comunitario, el cual, de acuerdo con la doctrina, se conforma de normas surgidas del derecho originario, el derecho complementario y el derecho derivado (art. 12 del Protocolo de Tegucigalpa; Mangas Martín, A. y D. J. Liñán Nogueras, 2016; Salazar Grande \& Ulate Chacón, 2009).

El derecho originario es el instituido por los tratados fundacionales de la comunidad; en el caso del SICA, cabe en esta categoría, el Protocolo de Tegucigalpa, sus reformas y modificaciones, el cual constituye la fuente primaria del derecho comunitario centroamericano. Este tratado establece las características, principios, objetivos, órganos, instituciones y las competencias que tiene el sistema institucional de integración (Guerrero Mayorga, 2008).

El derecho derivado o secundario es el que surge de los órganos e instituciones comunitarias, que aunque no tiene fuerza jurídica constituyente como el derecho originario y está limitado por reglas precisadas por los Estados, en el Tratado fundacional, por principio de atribución, posee poder normativo, por lo cual es de aplicación directa y de ejecución inmediata, sin que se requiera procedimiento de ratificación legislativa en cada Estado nación. En el caso del SICA, corresponde catalogar en este tipo de derecho, por ejemplo, las decisiones adoptadas por los Consejos de Ministros del SICA, ya sean reglamentos, resoluciones, acuerdos y recomendaciones (Arts. 7-12 del reglamento de para la adopción de decisiones del SICA de 2013) (Guerrero Mayorga, 2008).

Finalmente, el derecho complementario está integrado por aquellos tratados internacionales celebrados entre los Estados miembro de la comunidad en ámbitos no estrictamente comunitarios aunque con incidencia en los objetivos de la misma comunidad. También es complementario todo derecho surgido de los actos convencionales entre la Comunidad y terceros Estados y otras organizaciones internacionales. En sí, el derecho comunitario complementario se erige como una prolongación de los objetivos y propósitos definidos en el derecho originarios y sus modificaciones posteriores.

Para los efectos del tema que estamos tratando, resulta ser derecho complementario comunitario todo convenio y acuerdo suscrito por el SICA y sus Estados miembros, dentro de su institucionalidad o no, en materia de Derechos Humanos. Así en esta categoría caben, la declaración universal de los Derechos Humanos, así como también la Convención Americana de Derechos Humanos, por mencionar los principales del Derechos Internacional de los Derechos Humanos, pero también se incluiría el Convenio Constitutivo de la Comisión Centroamericana de medio ambiente y desarrollo, el Tratado Marco de Seguridad Democrática Centroamericana y el Tratado de Integración Social, por mencionar los más emblemáticos en el SICA.

Destacados estos elementos del derecho comunitario centroamericano, es importante poner de relieve la supremacía de la que gozan tanto los DDHH como el Derecho comunitario frente al Derecho interno de los Estados. Los DDHH se instituyen, por principio, en ius cogens, y están reconocidos con un especial rango constitucional. En virtud del principio pro homine deben aplicarse con preeminencia sobre toda norma para proteger mejor 
a la persona en su condición de ser humano, máxime si es una norma comunitaria vinculante para los Estados mismos, por trasladarse a un ámbito supranacional.

Esto queda reafirmado por el artículo 4 del protocolo de Tegucigalpa, el cual establece los principios identitarios del SICA, los cuales son:

* la tutela, respeto y promoción de los Derechos Humanos,

* la paz, la democracia y el desarrollo y libertad,

* la solidaridad centroamericana,

* la gradualidad, especificidad y progresividad del proceso de integración económica (Cláusula Centroamericana de Excepción);

* la globalidad del proceso de integración y la participación democrática, en él, de todos los sectores sociales;

* la seguridad jurídica de las relaciones entre los Estados Miembros y la solución pacífica de sus controversias;

* la buena fe de los Estados Miembros en el cumplimiento de sus obligaciones; y

* el respeto a los principios y normas de la Carta de la ONU y de la OEA, y las Declaraciones emitidas en las Reuniones Presidenciales Centroamericanas desde mayo de 1986.

\section{El rol de la CCJ en la tutela de los DDHH en Centroamérica}

Sobre la materia de derechos humanos, si bien el SICA no posee un catálogo de derechos fundamentales propio del Derecho Comunitario centroamericano, sí existen principios generales reconocidos por su tratado fundacional, conocido como Protocolo de Tegucigalpa (PT), cuya garantía de respeto es competencia de la Corte Centroamericana de Justicia (CCJ), institución regional de carácter supranacional, encargada también de la interpretación del Derecho comunitario en sus definiciones derivadas y complementarias para la salvaguardia de los propósitos y principios del SICA.

Por su parte, el artículo 12 del PT establece que la Corte Centroamericana de Justicia es uno de los órganos fundamentales del Sistema de la Integración Centroamericana SICA y "que garantizará el respeto del derecho, en la interpretación y ejecución del presente Protocolo y sus instrumentos complementarios o actos derivados del mismo". Además, en el Convenio de Estatuto de la CCJ, en el inciso segundo del primer artículo se le define como “... el Órgano Judicial principal y permanente del Sistema de la Integración Centroamericana, cuya jurisdicción y competencia regionales son de carácter obligatorio para los Estados".

Así también, los artículos 3 a. y 4 a. del PT establecen que es principio fundamental del SICA el irrestricto respeto, tutela y promoción de los derechos fundamentales. Los artículos 9 y 10 del mismo protocolo disponen que los órganos, organismos e instituciones del Sistema, entre ellos la CCJ, tienen la obligación imperativa y primaria de guiarse por los mismos propósitos y principios.

No obstante, el art. 25 del convenio de estatuto de la CCJ (de 1992 reformado en 2004), establece que la competencia de la CCJ no se extiende a la materia de derechos humanos, la cual corresponde exclusivamente a la Corte Interamericana de Derechos Humanos. Esto ha producido importantes discusiones en el momento de la admisión o no de determinados casos a ser sometidos al conocimiento de este Tribunal Regional y que están relacionados con supuestas violaciones a derechos humanos. 
Giammentei Avilés (2001), Guerrero Mayorga (2004) y Pérez-Cadalso Arias (2015) han coincidido en que, pese a esta exclusión de la competencia de la CCJ en materia de DDHH, cabe indicar los siguientes aspectos en que sí se puede admitir la actuación de la CCJ:

1. La exclusión se refiere a violaciones a DDHH cometidas por Estados que han reconocido la jurisdicción de la Corte IDH, pero no incluiría a los órganos, organismos e instituciones del SICA que fuesen señalados por cometer actos violatorios en el contexto y marco de aplicación del Derecho Comunitario Centroamericano.

2. Los actos que impliquen Derecho Derivado deben integrar la base del principio comunitario del respeto irrestricto a los derechos humanos. La tutela en estos actos le corresponde a la CCJ.

3. Los Acuerdos de Paz de Esquipulas I y II elevaron a nivel de Derecho Comunitario los principios que otorgan derechos al conglomerado social que integra la Comunidad Centroamericana, garantizando de manera particular para los ciudadanos derechos comunitarios individualizados, los cuales son oponibles a la actuación de los Estados que la conforman, cuando aquellos son afectados por sus decisiones o actos.

Sobre los derechos comunitarios individualizados, cabe destacar que en plena utilización del ius standi, la CCJ tiene competencia para conocer disputas entre personas naturales y los Estados o los órganos del sistema (art. 10 de la ordenanza de procedimientos de la CCJ).

De esta forma artículo 22 del Convenio de Estatuto de 1992 establece cuatro competencias en las que un individuo puede ser Parte de un proceso ante la CCJ:

c) Conocer, a solicitud de cualquier interesado, acerca de las disposiciones legales, reglamentarias, administrativas o de cualquier otra clase dictadas por un Estado, cuando afecten los Convenios, Tratados y cualquier otra normativa del Derecho de la Integración Centroamericana, o de los Acuerdos o resoluciones de sus órganos u organismos;

f) Conocer y resolver a solicitud del agraviado... cuando de hecho no se respeten los fallos judiciales;

g) Conocer de los asuntos que someta directa e individualmente cualquier afectado por los acuerdos del Órgano u Organismo del Sistema de la Integración Centroamericana;

j) Conocer en última instancia, en apelación, de las resoluciones administrativas, dictadas por los Órganos u Organismos del Sistema de la Integración Centroamericana, que afecten directamente a un miembro del personal del mismo y cuya reposición haya sido denegada.

Además, estos preceptos se constituyen en una especie de competencia indirecta (Herdocia, 2003, citado por Guerrero Mayorga, 2004) en materia de DDHH para la CCJ, respecto de las solicitudes de cumplimiento de fallos judiciales pasadas en Cosa Juzgada, en tanto tal acción por parte de un Estado, además de ir en contra de los principios del Derecho Comunitario centroamericano, transgrede a la vez la Convención Americana de Derechos Humanos en los asuntos de Garantías Judiciales, igualdad ante la Ley y la protección judicial, establecidos en los artículos 8, 24 y 25 de la misma convención americana.

Con lo descrito, aunado al principio kompetenz kompetenz (arts. 30 del Convenio de estatuto de 1992 y 8 de la Ordenanza de procedimientos de 2014) de la CCJ, se colige que este tribunal regional posee un claro marco de actuación en la tutela de los DDHH para casos 
específicos que no choquen con las competencias ya otorgadas a la Corte IDH del sistema interamericano.

\section{Jurisprudencia de la CCJ sobre su competencia en materia de DDHH}

Para ilustración, en su marco competencial, la CCJ ha emitido sentencias que dan cuenta de estos planteamientos, como son:

I. el Caso 11-1-8-2000, José Viguer Rodrigo contra el Órgano Judicial del Estado de Nicaragua, por irregularidades en el proceso y discriminación por razón de nacionalidad. La sentencia se emitió en octubre de 2000 y estimó por primera vez la tesis de que la CCJ sí puede conocer de violaciones a los derechos humanos en determinados casos y las esferas de competencia de la CCJ y la Corte IDH.

En la sentencia se consideró (I): “Que si las supuestas infracciones fueran atribuidas a un Órgano, Organismo o Institución del Sistema de la Integración Centroamericana (SICA), como consecuencia del incumplimiento de la Normativa que rige este Sistema, podrían tal vez llegar a ser del conocimiento de esta Corte, en atención a que uno de los pilares en que se fundamenta el Sistema de la Integración Centroamericana (SICA), es el irrestricto respeto, tutela y promoción de los Derechos Humanos, de conformidad con los artículos 3a y 4a del Protocolo de Tegucigalpa, que este Tribunal está en la obligación de salvaguardar y hacer efectivos a lo interno del Sistema, ya que dichos Órganos, Organismos e Instituciones no están sujetos a la jurisdicción de la Corte Interamericana de Derechos Humanos, y los afectados por ellos quedarían sin protección alguna." $Y$ en su parte resolutiva por unanimidad de votos declara "sin lugar lo solicitado en el referido escrito, por no ser de la competencia de este Tribunal, ya que lo expresado es atinente a supuestas violaciones de Derechos Fundamentales atribuidos a los Órganos encargados de Administrar Justicia en Nicaragua, Estado para el cual está vigente la Convención Interamericana de Derechos Humanos y como consecuencia su conocimiento puede caer bajo la competencia exclusiva de la Corte Interamericana de Derechos Humanos, de acuerdo con lo establecido en los artículos 44 y 61 numeral 2 de esa Convención".

el Caso 1-18-02-2010, Pablo Javier Pérez Campos, Diputado ante el Parlamento Centroamericano (PARLACEN) por la República de Panamá, en su calidad de demandante, y Gilberto Manuel Succari de co-demandante en contra del Estado de Panamá. La CCJ emitió sentencia el 20 de octubre de 2010 por la Demanda con Acción de Nulidad e Incumplimiento de la Normativa Comunitaria. La sentencia favoreció en términos generales a los actores.

Se consideró (IX) la definición del término de Derechos Comunitarios Individualizados y la base filosófica los Acuerdos de Paz de Esquipulas I y II, y se determinó "Que con los Acuerdos de Paz de Esquipulas I y II se establece un entramado de objetivos, principios, valores y normas que sustentan el actual proceso de integración regional centroamericana. Este conjunto de valores y normas tienen por objetivo garantizar la finalidad de la integración regional centroamericana: como es el bienestar común por medio del 
desarrollo, la paz, la justicia y la democracia. En ese sentido, la normativa comunitaria incorpora principios, siendo éstos rectores del actual marco jurídico del Sistema de la Integración Centroamericana y del actuar de los Estados Parte como de los Órganos, Organismos e Instituciones de dicho Sistema. Mismos que son elevados al nivel de Derecho Comunitario y por consiguiente otorgan derechos al conglomerado social que integra la Comunidad Centroamericana, garantizando de manera particular para los ciudadanos, derechos comunitarios individualizados, los cuales son oponibles contra el actuar de los órganos de la comunidad y de los Estados que la conforman, cuando los mismos sean afectados por sus decisiones y actos."

el Caso 8-7-05-2012, Octavio Bejerano Kant contra el Estado de Panamá, demanda por la adopción de actos violatorios de disposiciones contenidas en el Protocolo de Tegucigalpa y el Reglamento Interno del PARLACEN. Se emitió sentencia el 7 de mayo de 2014. El Considerando XI fundamentó: "Siendo el derecho a elegir y ser electo por sufragio universal, directo $\mathrm{y}$ secreto, un derecho fundamental contemplado en el instrumento constitutivo del Sistema de la Integración Centroamericana tal como es el Protocolo de Tegucigalpa a la Carta de la Organización de Estados Centroamericanos en su artículo 3 literal a) y estando recogido dicho derecho en el Tratado Constitutivo del Parlamento Centroamericano en sus artículos 2 y 6; ese derecho fundamental se constituye en un derecho comunitario individual, correspondiendo por ello a este tribunal conocer y fallar en el presente caso." En consecuencia, el fallo de mérito declaró: "que el Estado de Panamá ha violentado el Derecho Comunitario y en consecuencia ha incurrido en responsabilidad, quedando obligado a la indemnización correspondiente por los daños y perjuicios causados, los cuales deberán ser del conocimiento de la jurisdicción nacional".

\section{Resoluciones de la CCJ sobre el Derecho a un medio ambiente sano}

Hasta el momento hemos hecho mención del marco de actuación de la CCJ en la tutela de los DDHH individuales; sin embargo, debemos también tomar en consideración que, de acuerdo con los artículos 4 i. y 12 e. del Protocolo de Tegucigalpa, también es parte de la normativa comunitaria centroamericana, como principio fundamental y norma imperativa, "El respeto a los principios y normas de la Organización de las Naciones Unidas (ONU) y de la Organización de Estados Americanos (OEA)", con los que se abre un abanico de posibilidades inmensas en esta materia, lo que incluiría todas las obligaciones internacionales surgidas de las Conferencias, Cartas y Tratados que han sido auspiciados por estos organismos referidos al derecho a un Medio Ambiente sano.

Al respecto, podemos mencionar dos importantes precedentes de resoluciones emitidos por la CCJ:

I. Consulta que sobre la Situación Jurídica de la Alianza para el Desarrollo sostenible (ALIDES/1994), que formuló la Secretaría General del SICA, el 3 de abril de 1995: La consulta formulada por el entonces Secretario General del Sistema 
SICA, Dr. Roberto Herrera Cáceres, el 21 de abril de 1995. La ALIDES es una sentida aspiración comunitaria referida a la forma de implementar políticas de desarrollo regional que armonicen el actuar de los gobiernos, gobernantes y gobernados para la realización del bien común, individual y colectivo de todos los habitantes en la región. En el documento se establecen lineamientos o directrices del desarrollo sustentable y armónico con el medioambiente. La CCJ, al contestar la consulta realizada, cataloga el instrumento del ALIDES como derecho derivado de la competencias que el Protocolo de Tegucigalpa le confiere a la Reunión de presidentes, órgano supremo del SICA, haciéndolo plenamente vinculante para todos los Estados miembros (Giammattei Avilés, 2001).

II. Sentencia 12-06-12-2011: Asociación Foro Nacional de Reciclaje (FONARE) y la Fundación Nicaragüense para el Desarrollo Sostenible contra el Estado de Costa Rica. El caso de "la trocha" en el rio San Juan: Durante la ventilación del conflicto limítrofe entre Costa Rica y Nicaragua en el río San Juan, se conoció que Cota Rica construyó una trocha de 130 km de longitud a sólo 50 metros de las orillas del río. Dos organizaciones civiles ambientales demandan al estado de Costa Rica ante la CCJ por el presunto daño que la sedimentación y sequedad le ha causado al ecosistema en todo el sector de la ribera del río.

Lo conducente de la sentencia resuelve de la sieguiente manera: "Condénese al Estado de Costa Rica por haber incurrido en irresponsabilidad por los daños ecológicos y conexos en el río San Juan de Nicaragua, así como al ecosistema compartido".

En mi opinión, este es un interesante precedente de parte de la CCJ en la tutela de derechos colectivos y difusos de los ciudadanos centroamericanos.

\section{Conclusiones}

Es importante destacar el vínculo entre los derechos comunitarios individualizados y los derechos humanos reconocidos por los instrumentos internacionales clásicos. En este sentido, hay que advertir que, ante las infracciones en contra de los DDHH en el contexto del SICA, debe atribuirse competencia a un órgano, organismo o institución del SICA. Además, debemos tener en cuenta que la CCJ posee un marco de actuación en materia de DDHH con base en los principios comunitarios establecidos en el Protocolo de Tegucigalpa, sus instrumentos complementarios y los actos derivados. Para el caso de la Corte IDH, su marco de actuación surge de las competencias atribuidas en el Pacto de San José y la Declaración Americana de Derechos y Deberes del Hombre. Debemos siempre destacar que los Acuerdos de Paz de Esquipulas I y II plasmaron un nuevo concepto de "derechos" a favor de los ciudadanos centroamericanos, que se consideran derechos comunitarios individualizados sustentados en los principios, valores y normas que forman parte del espíritu de la norma comunitaria centroamericana.

En razón de la incorporación del Derecho comunitario complementario, los DDHH individuales internacionales son parte del sistema jurídico centroamericano, exigibles y tutelables por los órganos e instituciones del SICA, de conformidad con los artículos 9 y 10 del Protocolo de Tegucigalpa.

Los precedentes citados en materia de DDHH sobre un medio ambiente sano muestran que todavía hacen falta realizar mayores desarrollos jurisprudenciales, que permitan 
una acción coordinada entre la CCJ con otros órganos e instituciones del sistema y los Estados miembro, para que se garantice la efectividad de los fallos. La problemática del funcionamiento de jurisdicción de la Corte, en razón de la falta de ratificación por parte de todos los Estados miembro del SICA, constituye también un handicap en este cometido para lograr el fortalecimiento del Derecho comunitario.

Una actuación coordinada entre los órganos e instituciones del SICA, junto con los Estados miembro, la CCJ y los organismos promotores de DDHH, con la implementación de protocolos de actuación, permitiría la asunción de compromisos más sostenibles en beneficio de la garantía del goce de los derechos de los ciudadanos centroamericanos en el contexto de su proceso de integración.

\section{Referencias}

Giammattei Avilés, J. A. (2001). Los Derechos Fundamentales, la Corte Centroamericana de Justicia y la protección del medio ambiente. Recuperado de http://historico.juridicas.unam.mx/publica/librev/rev/dconstla/cont/2001/pr/pr13.pdf.

Guerrero Mayorga, O. (2004). “La Promoción y Protección de los derechos Humanos: Organizaciones y Mecanismos Internacionales: El Sistema Centroamericano de Protección de los Derechos Humanos". Revista de Derecho Facultad de Ciencias Jurídicas UCA (8) 89-100. Recuperado de https://www.lamjol.info/index.php/DERECHO/article/view/982.

Guerrero Mayorga, O. (2008). El Derecho comunitario: Concepto, naturaleza y principales diferencias con el derecho internacional y el derecho interno. En: "Jornadas de Derecho internacional" del 19 al 23 de noviembre de 2007, Universidad Centroamericana, Managua, Nicaragua. Organización de Estados Americanos.

Mangas Martín A. y D. J. Liñán Nogueras (2008). Instituciones y Derecho de la Unión Europea. Quinta Edición, TECNOS. Madrid, pp. 389-449.

Ordenanza de procedimientos de la CCJ de 2014

Pérez-Cadalso Arias, G (2015). "La tutela de los derechos humanos en el proceso de integración regional centroamericano". Recuperado de http://portal.ccj.org.ni/ccj/wp-content/uploads/LTDLDHIE.pdf

Protocolo de Tegucigalpa.

Reglamento para la adopción de decisiones del SICA del 2013.

Salazar Grande, C. E. \& E. N. Ulate Chacón, (2009). Manual de Derecho Comunitario Centroamericano. San Salvador: orbi.iure. 
